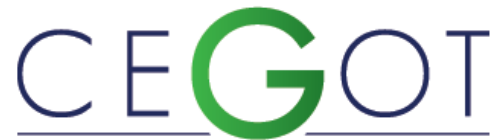

Centro de Estudos de Geografia e Ordenamento do Território

\author{
MOI, PAULA \\ Universidade Federal da Bahia, UFBA. \\ 40110-903, Salvador, Brasil (Avenida Reitor Miguel Calmon, s/n. Vale do Canela) \\ paulacpmoi@gmail.com \\ RibeiRo, AlEXANDRO \\ Universidade Federal de Mato Grosso, UFMT. \\ 78060-900, Cuiabá, Brasil. \\ ivalex_6@hotmail.com \\ ZAVALA, ARTURO \\ Universidade Federal de Mato Grosso, UFMT. \\ 78060-900, Cuiabá, Brasil \\ zavala.arturo@gmail.com \\ MOI, GISELE \\ Universidade Federal de Sergipe, UFS. \\ 49060-108, Aracaju, Brasil \\ gisele.pedroso.moi@gmail.com
}

\title{
Aspectos ecossocioeconômicos do agronegócio ao sul da Amazônia Legal
}

\section{Eco-socio-economic aspects of agribusiness on southern Legal Amazon}

Referência: Moi, Paula; Ribeiro, Alexandro; Zavala, Arturo; Moi, Gisele (2020). Aspectos ecossocioeconômicos do agronegócio ao sul da Amazônia Legal. Revista de Geografia e Ordenamento do Território (GOT), n.ㅇ 20 (Dezembro). Centro de Estudos de Geografia e Ordenamento do Território, p. 57-78, dx.doi.org/ 10.17127/got/2020.20.003

\section{RESUMO}

Pretende-se com esta pesquisa analisar a distribuição espacial conjunta do nível de especialização agropecuária, entre 2005 a 2014, e explorar possíveis associações com aspectos ambientais, sociais e econômicos. Sendo assim, foi possível analisar se a especialização do setor agropecuário no Estado de Mato Grosso - maior produtor e exportador de commodities brasileiro e localizado ao sul da Amazônia Legal - está associada a uma economia sustentável. Para isso, foi utilizada uma abordagem econométrica espacial compreendendo várias análises de estatística espacial: quociente locacional (QLargo), Índice de Moran I e análise de regressão múltipla espacial. Constatou-se que o agronegócio matogrossense é sustentado por um modelo agroquímico-dependente consolidado que desfavorece a ecossocioeconomia da especialização agropecuária, atividade esta que afeta o bem-estar social e o meio ambiente.

Palavras-chave: Ecossocioeconomia; Agropecuária; Análise de cluster; Análise espacial.

\section{ABSTRACT}

This research aims to analyze the joint spatial distribution of the level of agricultural specialization, between 2005 and 2014, and explore possible associations with 
environmental, social and economic aspects. Thus, it was possible to analyze whether the specialization of the agricultural sector in the State of Mato Grosso - the largest producer and exporter of commodities in Brazil and located in the south of the Legal Amazon - is associated with a sustainable economy. For this, a spatial econometric approach was used, comprising locational quotient (QLargo), Moran I index and multiple spatial regression analysis. It was found that agribusiness in Mato Grosso is supported by a consolidated agrochemical-dependent model that disadvantages the eco-socio-economics of the agricultural specialization, an activity that affects welfare state and the environment.

Key words: Eco-socio-economics, Agricultural; Cluster analysis; Spatial analysis.

\section{Introdução}

A atividade agropecuária é desenvolvida no espaço rural, em áreas que se encontram utilizadas pelo setor primário da economia. Esse espaço é caracterizado pela presença de cobertura vegetal original, animais silvestres, áreas antropizadas não produtivas, além de também cultivar recursos ou extraí-los da natureza para posterior consumo ou transformação em mercadorias. Em síntese, a produção no espaço rural é composta pela agropecuária - expressão utilizada para designar de forma agrupada a pecuária e a agricultura (Marafon \& Lima, 2018).

Nesse contexto, o nível de especialização produtiva agropecuária afeta a maneira de se produzir e também o relacionamento entre parceiros da cadeia produtiva, além de transfigurar o espaço geográfico onde essas atividades estão concentradas. A teoria prática do desenvolvimento de Ignacy Sachs sugere que somente por meio de uma ecossocioeconiomia é possível atingir o desenvolvimento, pois permite associar o sucesso econômico, ao aumento democrático do bem-estar social e a conservação ambiental (Sachs, 2007).

De acordo com os dados disponíveis no Sistema IBGE de Recuperação Automática - SIDRA (IBGE, 2020a), em 2015, a participação do Valor Adicionado Bruto da Agropecuária (VABagro) no Valor Adicionado Bruto Total (VAB) brasileiro equivalia a 5,09\%. Entretanto, avaliando esses números em uma escala regional, percebe-se que a participação do VABagro em relação ao VAB na Região Norte do Brasil fica mais expressiva (10,64\%), seguida da Região Centro-Oeste (9,69\%) e Sul (8,41\%). 
Ao sul da Amazônia Legal, localiza-se o estado de Mato Grosso de grande extensão territorial, localizado na região Centro-Oeste do Brasil e organizado em 22 microrregiões, 5 mesorregiões, e 141 municípios (Figura 1).

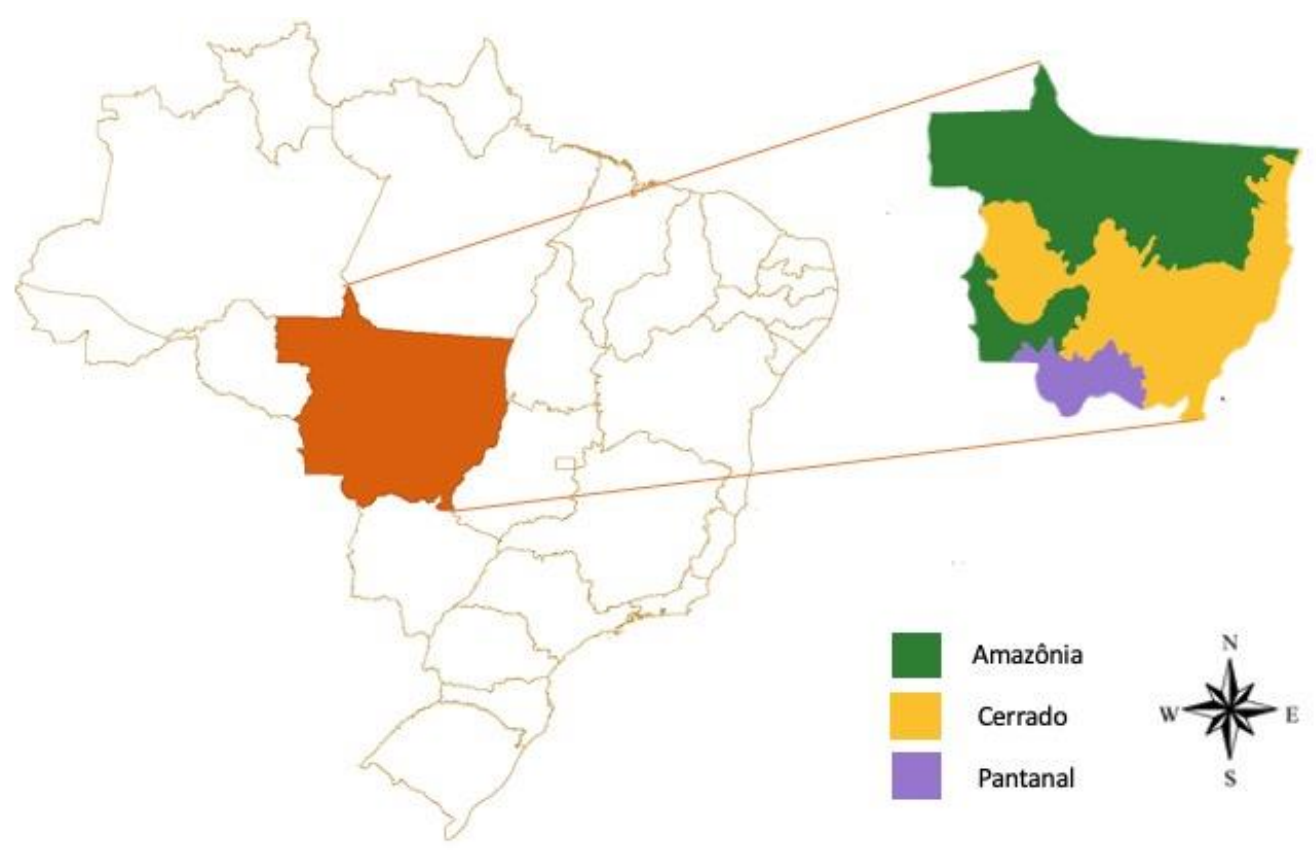

Figura 1 - Localização de Mato Grosso no Brasil e suas respectivas áreas de abrangência dos biomas. Fonte: Elaborado com o auxílio do Software GeoDa 1.14.0.4 por meio dos dados disponibilizados pelo IBGE, 2020.

Além disso, essa região abriga três dos grandes biomas brasileiros: Amazônia (53\%), Cerrado (40\%) e Pantanal (7\%). Por outro lado, se destaca por ser o maior estado brasileiro produtor de soja, milho e algodão herbáceo, segundo maior produtor de arroz e quarto maior produtor de cana-de-açúcar, além de liderar o ranking entre os estados brasileiros que mais consomem agrotóxicos (IBGE, 2020).

O Bioma Cerrado, em especial, onde a moderna agricultura de cunho produtivista se instaurou intensivamente desde meados da década de 1970, se tornou a "vaca sagrada" da economia nacional. Por esse motivo, os estudos teóricos que envolvem os cerrados brasileiros e a moderna agricultura não são uma missão simples (Santos, 2015).

Nesse contexto, os municípios do estado de Mato Grosso possuem sua economia intimamente atrelada ao agronegócio, cujos aspectos ecossocioeconômicos ainda não são contemplados dentro da política de avaliação do desenvolvimento brasileiro. Essa situação 
sugere o questionamento referente a especialização do setor agropecuário no Estado de Mato Grosso e sua associação a uma economia sustentável.

Apesar das discussões em diversos campos do conhecimento envolverem a concepção do conceito de sustentabilidade, elas, inevitavelmente, se apoiarão em duas doutrinas científicas: economia e ecologia (Veiga, 2010). Deste modo, esse estudo se baseia nos preceitos da economia ambiental que "aplica aos problemas ecológicos as ferramentas da economia neoclássica"(Cavalcanti, 2010, pp. 60-61).

Nesse sentido, o objetivo deste estudo foi investigar a distribuição espacial conjunta do nível de especialização na atividade agropecuária dos municípios do estado de Mato Grosso, no período compreendido entre 2005-2014, e explorar possíveis associações com os aspectos sociodemográficos, econômicos e ambientais. Esse período foi escolhido em detrimento da disponibilidade e combinação dos dados em todas as dimensões estudadas: econômicas, sociais e ambientais.

\section{Métodos}

Este estudo observacional, exploratório e transversal incluiu dados secundários dos municípios do estado de Mato Grosso que se encontram disponíveis livremente nas bases de dados brasileiras.

Como aspectos metodológicos foram considerados o coeficiente de correlação de Pearson, Quociente Locacional e Índice de Moran, com o objetivo de identificar associações entre variáveis, assim como as associações das variáveis e as localidades geográficas.

Quando se fala de variáveis unidimensionais, Taylor (1990) afirma que o coeficiente de correlação linear (Correlação de Pearson) representa o grau de associação linear entre duas variáveis em estudo.

Quando se deseja pesquisar concentração, Lara et al. (2010) afirmam que o quociente locacional é um coeficiente de concentração e serve para expressar qual região é mais importante em relação a seus setores produtivos.

Quando se deseja entender as relações espaciais, Celemín (2009), afirma que a autocorrelação espacial surge como a forma de estudar a propagação de um determinado 
fenômeno no espaço geográfico, uma forma de identificar esta propagação é mediante o índice de Moran, que permite relacionar em forma conjunta a dependência entre os lugares geográficos e as variáveis em estudo, com o objetivo da identificação espacial dos fenômenos econômicos-sociais.

Para isto, foi utilizada uma abordagem econométrica espacial que inicialmente determinou a especialização produtiva agropecuária pela metodologia do quociente locacional (QLargo). Após isso, foi investigada a autocorrelação espacial desse QLagro por meio do Índice de Moran I. Para a análise bivariada e análise de regressão múltipla espacial as variáveis foram distribuídas em três dimensões - ambientais, sociais e econômicas.

\subsection{Quociente Locacional (QL)}

Com o intuito de definir o nível de especialização na atividade agropecuária dos 141 municípios de Mato Grosso no período de 2005 a 2014, foi necessário determinar a média da participação percentual do Valor adicionado bruto a preços correntes da agropecuária (VABagro) municipal e estadual para o período em análise, tabulados a partir do Sistema de Contas Nacionais (SCN), disponíveis no Sistema IBGE de Recuperação Automática - SIDRA (IBGE, 2010). Para a determinação do QLagro municipal se utilizará a seguinte fórmula:

$$
\text { QLagro }=\frac{\frac{\text { VABagro municipal }}{\text { VABagro estadual MT }}}{\frac{\text { PIB municipal }}{\text { PIB Estadual MT }}}(1)
$$

A partir do tratamento destes dados e por meio do cálculo do Quociente Locacional foi obtida variável dependente do estudo: Grau de especialização na atividade agropecuária municipal (QLagro). Este indicador representa a relação entre a participação percentual de um município em um setor particular, que neste caso será o agropecuário, e a participação do município em questão na produção total da economia estadual (Haddad, 1989). Com este indicador, pode-se determinar se um dado município é muito ou pouco especializado na atividade agropecuária. Os parâmetros de referência para a interpretação deste indicador são (Crocco, Galinari, Santos, Lemos, \& Simões, 2006): QL > 1 o município é especializado na atividade agropecuária da sua região de referência, $Q L<1$ o município não tem a sua produção interna especializada neste setor e $Q L=1$ indica que a concentração da atividade econômica na região é igual à concentração da mesma no estado. 
Existem algumas outras maneiras de se identificar as características das aglomerações produtivas além da metodologia do Quociente Locacional (QL) que mensura a especialização de dado setor, como: o Índice de Participação Relativa (PR) que identifica a importância da atividade no setor estudado; e o Índice de Concentração Normalizado (ICn), que é uma combinação do QL com o PR (Lins \& Da Silva, 2017). Portanto, o Quociente Locacional foi o método que atendeu ao objetivo desta pesquisa, que primeiramente visa identificar o nível de especialização econômica na atividade agropecuária. Além disso, quando avaliamos essa especialização econômica com base no número de empresas existentes em determinada região, estamos trabalhando num contexto microeconômico, mas quando pesquisamos a nível municipal estamos trabalhando uma lógica macroeconômica. Portanto, as restrições de Crocco et al. (2006), quanto a utilização do QL, não se ajustam a este trabalho.

\subsection{Estatística de Moran}

Partindo para o modelo analítico dos dados, inicialmente foi efetuada a análise exploratória univariada dos dados espaciais para investigação de autocorrelação espacial global e local do QLagro dos municípios mato-grossenses através do índice I de Moran, sob as suposições de normalidade e de randomização.

A distribuição de valores do índice de Moran Global varia entre $-1,0$ e $+1,0$ e testa se áreas conectadas apresentam maior semelhança quanto ao indicador estudado, do que seria esperado num padrão aleatório (Anselin \& Rey, 2010). Para o cálculo utilizou-se o peso tipo Queen. O índice varia de $-1<I<1$ e tem média $E(I)=[1 / n-1]$. A estatística proporcionará observar se os dados analisados apresentam ou não homogeneidade, e se apresentarem, consequentemente existirá correlação espacial.

Ocasionalmente, pode ocorrer que esta estatística global esteja sendo influenciada por resultados locais, sendo conveniente que se faça uma análise local. Para isto, utilizou-se a análise do Índice de Moran Local ou LISA - Local Indicator of Spatial Autocorrelation (Anselin, 1995), que contribuiu para identificar a associação espacial do QLagro dos municípios do estado (Almeida, 2004). Nesse sentido, podem ser observados quatro casos de agrupamentos espaciais: 
1. Alto-Alto (High-High): os municípios que compõem este agrupamento e também seus vizinhos, apresentam valores altos para a variável em estudo, ou seja, acima da média;

2. Baixo-Baixo (Low-Low): os municípios deste agrupamento e seus vizinhos, apresentam valores baixos para a variável em estudo;

3. Alto-Baixo (High-Low): o agrupamento espacial apresenta valores alto, mas os valores da variável em estudo nos municípios circunvizinhos são baixos;

4. Baixo-Alto (Low-High): o agrupamento espacial apresenta valores baixo referente a variável em estudo, mas à mesma apresenta valores altos nos municípios circunvizinhos.

Os índices Global e Local de Moran foram calculados pelo software livre GeoDa 0.95-i.

\subsection{Coeficiente de correlação de Pearson (r)}

Foi necessário avaliar conjuntamente os indicadores associados ao QLagro, por meio do coeficiente de correlação de Pearson ( $r$ ) com o auxílio do software SPSS 18.0, que neste estudo foram agrupados em três categorias: variáveis sociodemográficas, econômicas e ambientais. Sendo assim, as variáveis independentes selecionadas para o estudo foram recuperadas de acordo com a tabela 1.

Foram selecionadas para a análise de regressão múltipla espacial aquelas variáveis cujo nível descritivo de teste apresentou p $<0,20$ (Moi, Silva, Galvão, de Castro Meneghim, \& Pereira, 2018). Por fim, o próximo passo foi realizar a análise de regressão múltipla espacial. 
Tabela 1 - Origem das variáveis do estudo.

\begin{tabular}{|c|c|c|}
\hline Codificação & Variável & Fonte \\
\hline \multicolumn{3}{|c|}{ Ambientais } \\
\hline TXDESF & Taxa de área Desflorestada & INPE/PRODES \\
\hline TXHIDROG & Taxa da área correspondente a hidrografia & INPE/PRODES \\
\hline CAGROTO & Consumo de Defensivos Agrícolas & IBGE - Censo Agropecuário 2006 \\
\hline \multicolumn{3}{|c|}{ Econômicas } \\
\hline IMPOS & $\begin{array}{l}\text { Impostos, líquidos de subsídios, sobre produtos, a preços } \\
\text { correntes }\end{array}$ & $\begin{array}{l}\text { IBGE - Sistema de Contas } \\
\text { Nacionais }\end{array}$ \\
\hline TEAGROP & $\begin{array}{l}\text { Número de empresas da Agricultura, pecuária, produção } \\
\text { florestal, pesca e aquicultura }\end{array}$ & $\begin{array}{l}\text { IBGE - Cadastro Central de } \\
\text { Empresas }\end{array}$ \\
\hline TINDEXT & Taxa de empresas da Indústria extrativista & $\begin{array}{l}\text { IBGE - Cadastro Central de } \\
\text { Empresas }\end{array}$ \\
\hline TETRANP & $\begin{array}{l}\text { Proporção de empresas ligadas ao setor de transporte, } \\
\text { armazenagem e correio }\end{array}$ & $\begin{array}{l}\text { IBGE - Cadastro Central de } \\
\text { Empresas }\end{array}$ \\
\hline TEAGESG & $\begin{array}{l}\text { Taxa de empresas água, esgoto, atividades de gestão de } \\
\text { resíduos e descontaminação }\end{array}$ & $\begin{array}{l}\text { IBGE - Cadastro Central de } \\
\text { Empresas }\end{array}$ \\
\hline RSMED & Renda média domiciliar per capita & DATASUS \\
\hline NCRURAL & $\begin{array}{l}\text { Número de estabelecimentos que obtiveram acesso ao } \\
\text { crédito rural }\end{array}$ & IBGE - Censo Agropecuário 2006 \\
\hline VCRURAL & Valor dos financiamentos agropecuários obtidos & IBGE - Censo Agropecuário 2006 \\
\hline MAQIMP & $\begin{array}{l}\text { Número de máquinas e implementos agrícolas existentes } \\
\text { nos estabelecimentos agropecuários }\end{array}$ & IBGE - Censo Agropecuário 2006 \\
\hline TUQTRANSP & Taxa de utilização de meios de transportes & IBGE - Censo Agropecuário 2006 \\
\hline \multicolumn{3}{|c|}{ Sociodemográficas } \\
\hline TPOPURB & Taxa de população residente urbana & IBGE - Censo Demográfico 2010 \\
\hline TPOPRUR & Taxa de população residente rural & IBGE - Censo Demográfico 2010 \\
\hline ANALFA & $\begin{array}{l}\text { Nível de instrução da pessoa que dirige o estabelecimento } \\
\text { - Analfabeto }\end{array}$ & IBGE - Censo Agropecuário 2006 \\
\hline TDESEMP & $\begin{array}{l}\text { Taxa de desemprego: Percentual da população de } 16 \text { anos } \\
\text { e mais, economicamente ativa, desocupada. }\end{array}$ & DATASUS \\
\hline IDHM & O Índice de Desenvolvimento Humano Municipal. & IPEA \\
\hline GINI & Índice Gini & $\begin{array}{l}\text { Altas de Desenvolvimento } \\
\text { Humano do Brasil }\end{array}$ \\
\hline SANINAD & Proporção de domicílios com saneamento inadequado & $\begin{array}{l}\text { Altas de Desenvolvimento } \\
\text { Humano do Brasil }\end{array}$ \\
\hline TOBNAT & Taxa de Óbitos Naturais & $\begin{array}{l}\text { IBGE - Estatísticas do Registro } \\
\text { Civil }\end{array}$ \\
\hline TOBVIOL & Taxa de Óbitos Violentos & $\begin{array}{l}\text { IBGE - Estatísticas do Registro } \\
\text { Civil }\end{array}$ \\
\hline IFDM & Evolução do Índice Firjan & Sistema Firjan \\
\hline
\end{tabular}

Fonte: Elaborado pelos autores (2020).

\subsection{Análise de Regressão Múltipla Espacial}

A análise de regressão múltipla espacial possui os mesmos pressupostos da regressão linear tradicional, porém leva em consideração as tendências ou correlações espaciais dos dados e, caso seja constatada autocorrelação espacial, são incorporados determinados parâmetros que possibilitam a retirada destes efeitos. A qualidade do ajuste do modelo de regressão 
espacial também é semelhante à do modelo de regressão múltipla tradicional, sendo verificada por meio da análise de resíduos e também com base no índice de Moran (Anselin \& Rey, 2010). Para esta análise foi utilizado o método Backward, que incorpora inicialmente todas as variáveis coletadas e, depois disso, exclui-se as variáveis por etapas, seguindo os seguintes critérios estabelecidos:

1. Inclusão da variável que, por meio da aplicação do coeficiente de correlação de Pearson ( $r$ ), apresentou $\mathrm{p}<0,20$; esta inclusão não impede que também sejam incluídas "variáveis de ajuste" independente do grau de associação com a variável dependente.

2. Exclusão das variáveis com o $\mathrm{p} \geq 0,05$, iniciando-se a exclusão pela variável que obteve o maior $p$-valor.

Na verificação dos pressupostos da regressão linear, foram efetuadas análises gráficas entre resíduos padronizados, valores observados e preditos, além do diagnóstico de normalidade, por meio de gráficos Q-Q plot. Também foram aplicados os pós-testes de Breusch-Pagan e Koenker-Bassett para verificação de heterocedasticidade. Finalmente, na obtenção de modelos finais, também foi verificada a não autocorrelação espacial dos resíduos. 0 software utilizado para essa análise foi o GeoDa 0.95-i.

\section{Resultados}

A distribuição espacial do nível de especialização na atividade agropecuária (QLagro) no estado de Mato Grosso, no período de 2005-2014, de acordo com o quartil está ilustrada na Figura 2. Dentre os 141 municípios, 106 foram identificados como especializados na atividade agropecuária (75,18\%). Observa-se que as taxas mais altas referentes ao QLagro ocorreram na região Central e Sudeste (QLagro $\geq 3$ ).

Importante salientar, que os municípios com os maiores índices de especialização agropecuária, destacados na figura 2 , são relativamente novos, entre 20 e 80 anos de emancipação. 


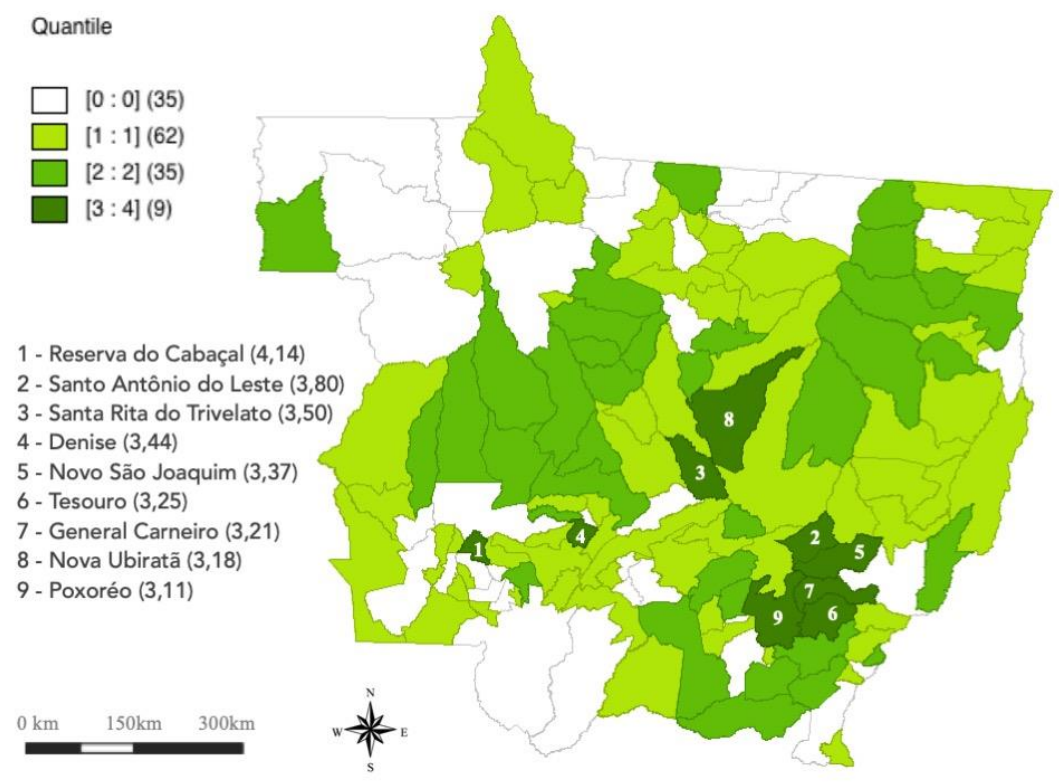

Figura 2 - Nível de especialização agropecuária de Mato Grosso entre 2005-2014, de acordo com quartil.

Fonte: resultados da pesquisa (2020).

Foi possível observar que a distribuição espacial global da especialização na atividade agropecuária não foi aleatória (Figura 3), resultando em uma autocorrelação/dependência espacial positiva ( $(=0.0507897$; $p$-valor $=0,001$ para 999 permutações).

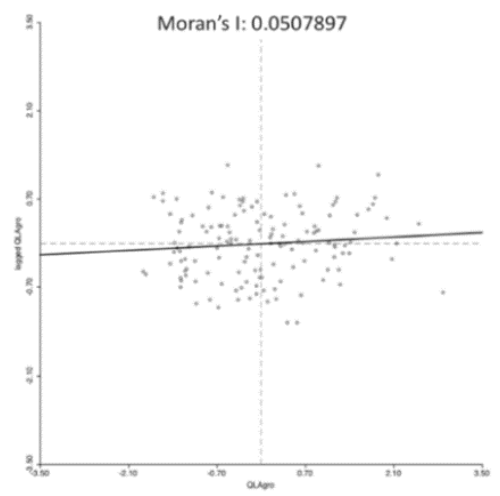

Figura 3 - Scatter Plot Frame: Diagrama de espelhamento de Moran I.

Fonte: resultados da pesquisa (2020).

Neste sentido, este resultado significa que os polígnos são similares em localização e atributos. Decompondo este índice global, podemos visualizar na forma de mapas os resultados obtidos para a Estatística de Moran Local para o nível de especialização agropecuária de Mato Grosso entre 2005-2014 (Figura 4). 


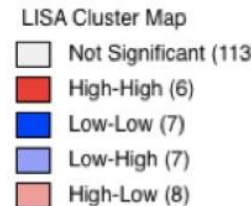

1 - Rondolândia

2- Cotriguaçu

3 - Novo Mundo

4 - Castanheira

5 - Nova Monte Verde

6 - Terra Nova do Nor

7 - Nova Maring

8 - Itanhangá

9 - Sorriso

10 - Sinop

11 - Feliza Natal

12 - Paranating

13 - Campinápolis

14 - Barra do Garças

15 - Tesouro

16 - Guiratinga

17 - Rondonópolis

18 - Poxoréu

19 - Primavera do Leste

20 - Acorizal

21 - Jangada

22 - Nossa Senhora do Livramento

23 - Lambrai D'Oeste

24 - Reserva do Cabaçal

25 - Vale de São Domingos

26 - Indiavaí

27 - Glória D'Oeste

28 - Porto Esperidião
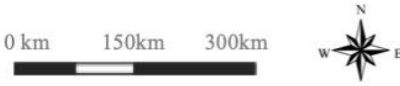

LISA Significance Map

$\square$ Not Significant (113

$p=0.05(24)$

$p=0.01(3)$

$p=0.001$

$\begin{array}{ll}\text { 1 - Rondolândia } & 15 \text { - Tesouro } \\ \text { 2- Cotriguaçú } & 16 \text { - Guiratinga } \\ \text { 3- Novo Mundo } & 17 \text { - Rondonópolis } \\ \text { 4- Castanheira } & 18 \text { - Poxoréu } \\ \text { 5- Nova Monte Verde } & 19 \text { - Primavera do Leste } \\ \text { 6- Terra Nova do Norte } & 20 \text { - Acorizal } \\ \text { 7- Nova Maringá } & 21 \text { - Jangada } \\ \text { 8- Itanhangá } & 22 \text { - Nossa Senhora do Livr } \\ \text { 9- Sorriso } & 23 \text { - Lambrai D’Oeste } \\ \text { 10- Sinop } & 24 \text { - Reserva do Cabaçal } \\ \text { 11- Feliza Natal } & 25 \text {-Vale de São Domingos } \\ \text { 12- Paranatinga } & 26 \text { - Indiavaí } \\ \text { 13-Campinápolis } & 27 \text { - Glória D'Oeste } \\ \text { 14- Barra do Garças } & 28 \text { - Porto Esperidião }\end{array}$

14 - Barra do Garças 28 - Porto Esperidião

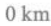

$150 \mathrm{~km} \quad 300 \mathrm{~km}$

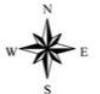

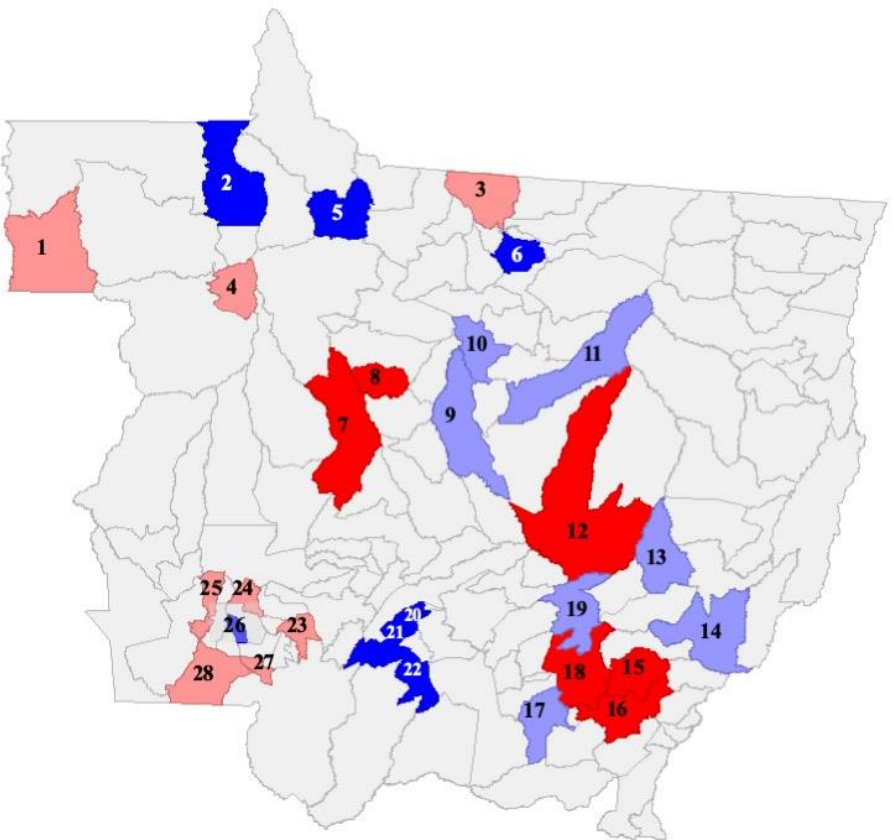

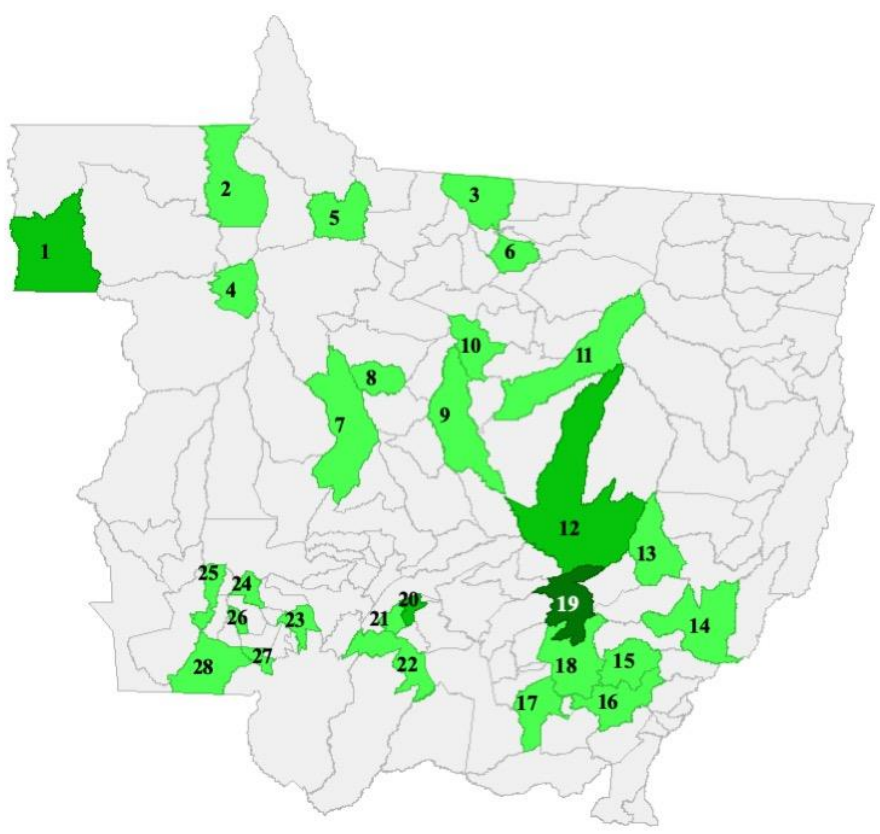

Figura 4 - Indicador de Moran Local para o nível de especialização agropecuária de Mato Grosso entre 2005-2014.

Fonte: resultados da pesquisa (2020).

O primeiro mapa, apresentado na figura 4 (Lisa Cluster Map), demonstra a ocorrência de autocorrelações espaciais do tipo "alto-alto" (High-high), indicando um agrupamento de municípios mato-grossenses com QLagro maiores: Nova Maringá, Itanhangá, Paranatinga, Tesouro, Guiratinga e Poxoréo. O que significa que os municípios que compõem estes agrupamentos e também seus vizinhos, apresentam valores altos para a variável em estudo, ou seja, acima da média. A ocorrência de autocorrelações espaciais do tipo "baixo-baixo" 
(Low-low), considera um grupo com taxas mais baixas, representada pelos municípios de Cotriguaçú, Nova Monte Verde, Terra Nova do Norte, Acorizal, Jangada, Indiavaí e Nossa Senhora do Livramento. Sendo assim, os municípios que compõem este agrupamento e também seus vizinhos, apresentam valores abaixo da média para a variável em estudo. Logo, os municípios de Rondolândia, Novo Mundo, Castanheira, Lambari D’Oeste, Reserva do Cabaçal, Vale de São Domingos, Glória D’Oeste e Porto Esperidião foram categorizados como pertencentes ao grupo "alto-baixo" (High-low), cenário em que a cidade ou o agrupamento espacial apresentam valores altos, mas os valores da variável em estudo (QLagro) nos municípios circunvizinhos são baixos. Já, os municípios de Sorriso, Sinop, Feliz Natal, Campinápolis, Barra do Garças, Rondonópolis e Primavera do Leste foram identificados como "baixo-alto" (Low-high), significando que o município ou o agrupamento espacial apresentam valores baixos referente ao nível de especialização agropecuária, mas esta especialização apresenta valores altos nos municípios circunvizinhos.

O segundo mapa, apresentado na figura 4 (Lisa Significance Map), identificou as características que predominam em agrupamentos significativos dentre os valores dos seus vizinhos. Nesse sentido, os municípios com níveis de especialização mais significativos foram Primavera do Leste ( $p=0,001)$; seguido por Acorizal, Rondolândia e Paranatinga $(p=0,01)$. 0 significado desse indicador no período em análise implica que existem externalidades multidirecionais dos níveis de especialidade na atividade agropecuária em alguns municípios de Mato Grosso, evidenciando que esses municípios têm dinâmica espacial própria.

$\mathrm{Na}$ análise bivariada (Tabela 2) foram selecionadas para a análise de regressão múltipla espacial aquelas variáveis cujo $\mathrm{p}<0,20$, para se obter um maior número de variáveis para a elaboração da regressão múltipla espacial, sem considerar aquelas variáveis que realmente não afetam o modelo. 
Tabela 2 - Matriz de correlação entre o QLagro e as variáveis selecionadas nos diferentes blocos de análise de Mato Grosso entre 2005-2014.

\begin{tabular}{|c|c|c|c|c|c|c|c|c|c|c|c|c|c|c|c|c|c|c|c|c|c|c|c|c|}
\hline & $\begin{array}{c}\text { QLAGR } \\
0 \\
\end{array}$ & TDESF & $\begin{array}{c}\text { THIDR } \\
\text { OG }\end{array}$ & $\begin{array}{c}\text { CAGRO } \\
\text { TO }\end{array}$ & $\begin{array}{c}\mathrm{IMPO} \\
\mathrm{S}\end{array}$ & $\begin{array}{c}\text { TEAGR } \\
\text { OP }\end{array}$ & $\begin{array}{c}\text { TINDE } \\
\mathrm{XT} \\
\end{array}$ & $\begin{array}{c}\text { TEAGE } \\
\text { SG } \\
\end{array}$ & $\begin{array}{c}\text { TETR } \\
\text { NP } \\
\end{array}$ & $\begin{array}{c}\text { RSME } \\
\text { D }\end{array}$ & $\begin{array}{c}\text { NCRRUR } \\
\text { AL }\end{array}$ & $\begin{array}{c}\text { VCRRUR } \\
\text { AL }\end{array}$ & $\begin{array}{c}\text { MAQI } \\
\text { MP }\end{array}$ & $\begin{array}{c}\text { TUQTRAN } \\
\text { SP } \\
\end{array}$ & $\begin{array}{c}\text { TPOPU } \\
\text { RB } \\
\end{array}$ & $\begin{array}{c}\text { TPOPR } \\
\text { UR }\end{array}$ & $\begin{array}{c}\text { ANALF } \\
\text { A }\end{array}$ & $\begin{array}{c}\text { TDESE } \\
\text { MP }\end{array}$ & IDHM & GINI & $\begin{array}{c}\text { SANIN } \\
\text { AD } \\
\end{array}$ & $\begin{array}{c}\text { TOBN } \\
\text { AT } \\
\end{array}$ & $\begin{array}{c}\text { TXOBVI } \\
\text { OL }\end{array}$ & $\begin{array}{c}\text { IFD } \\
M \\
\end{array}$ \\
\hline QLAGRO & 1 & & & & & & & & & & & & & & & & & & & & & & & \\
\hline TDESF & $-0,128$ & 1 & & & & & & & & & & & & & & & & & & & & & & \\
\hline THIDROG &,$- 178^{*}$ & $-0,13$ & 1 & & & & & & & & & & & & & & & & & & & & & \\
\hline CAGROTO & $\stackrel{-}{-269^{* *}}$ & ,186* & 0,108 & 1 & & & & & & & & & & & & & & & & & & & & \\
\hline IMPOS & $\begin{array}{c}- \\
, 265^{* *}\end{array}$ & $\begin{array}{c}- \\
0,123\end{array}$ & $-0,05$ & 0,062 & 1 & & & & & & & & & & & & & & & & & & & \\
\hline TEAGROP & 0,165 & $\underset{*}{231^{*}}$ & 0,026 & $-0,081$ & 0,094 & 1 & & & & & & & & & & & & & & & & & & \\
\hline TINDEXT & 0,008 & $\begin{array}{c}- \\
0,001\end{array}$ & 0,164 & 0,037 & $\begin{array}{c}- \\
0,073\end{array}$ & 0,014 & 1 & & & & & & & & & & & & & & & & & \\
\hline TEAGESG & 0,011 & 0,033 & 0,069 & $-0,019$ & $\overline{-}-\overline{0} 1$ & ,181* & $-0,113$ & 1 & & & & & & & & & & & & & & & & \\
\hline TETRNP &, $232^{* *}$ & 0,034 & $-0,148$ & 0,015 & $0, \overline{002}$ & $-0,083$ & $-0,074$ & 0,085 & 1 & & & & & & & & & & & & & & & \\
\hline RSMED & $-0,114$ & $\begin{array}{c}- \\
0,001\end{array}$ & $-0,143$ &, $256^{* *}$ & $\begin{array}{c}, 477^{*} \\
*\end{array}$ & 0,006 & $-0,096$ & $-0,146$ & $\begin{array}{c}, 251^{*} \\
*\end{array}$ & 1 & & & & & & & & & & & & & & \\
\hline $\begin{array}{c}\text { NCRRURA } \\
L\end{array}$ & $\begin{array}{c}- \\
, 274^{* *}\end{array}$ & 0,126 & 0,118 & ,607** & 0,033 & $-0,05$ & $-0,045$ & 0,065 & 0,044 & ,206* & 1 & & & & & & & & & & & & & \\
\hline $\begin{array}{c}\text { VCRRURA } \\
\text { L }\end{array}$ & 0,146 & $\begin{array}{c}- \\
0,113\end{array}$ & $-0,049$ & 0,106 & 0,075 & 0,038 & 0,05 & $-0,063$ & 0,096 & $\begin{array}{c}308^{*} \\
*\end{array}$ &, $227^{* *}$ & 1 & & & & & & & & & & & & \\
\hline MAQIMP & 0,1 & $\overline{-}^{-} 200^{*}$ & $-0,065$ & , 334** & ,198* & 0,087 & $-0,072$ & $-0,031$ & ,196* & $\begin{array}{c}, 577^{*} \\
*\end{array}$ &, $418^{* *}$ &, $456^{* *}$ & 1 & & & & & & & & & & & \\
\hline $\begin{array}{l}\text { TUQTRAN } \\
\text { SP }\end{array}$ & 0,157 & 0,042 & $-0,138$ & $-0,055$ & $\begin{array}{c}- \\
0,115\end{array}$ & 0,147 & $-0,121$ &,$- 205^{*}$ & 0,133 & $\begin{array}{c}316^{*} \\
*\end{array}$ & $-0,094$ & ,175* & ,296** & 1 & & & & & & & & & & \\
\hline TPOPURB & $\begin{array}{c}- \\
, 265 * *\end{array}$ & $\begin{array}{c}- \\
0,032\end{array}$ & $-0,054$ & 0,029 & $\begin{array}{c}, 312^{*} \\
* \\
-\end{array}$ & $-0,11$ & $-0,127$ & $-0,082$ & 0,076 & $\begin{array}{c}, 581^{*} \\
* \\
-\end{array}$ & 0,012 & 0,047 & ,335** & 0,142 & 1 & & & & & & & & & \\
\hline TPOPRUR &, $265 * *$ & 0,032 & 0,054 & $-0,029$ & $\stackrel{312^{*}}{*}$ & 0,11 & 0,127 & 0,082 & $-0,076$ & $\begin{array}{l}, 581^{*} \\
* \\
-\end{array}$ & $-0,012$ & $-0,047$ &, $335 *$ & $-0,142$ & $\begin{array}{c}1,000^{*} \\
*\end{array}$ & 1 & & & & & & & & \\
\hline ANALFA &,$- 211^{*}$ & ,207* & ,196* & $-0,044$ & $-\overline{-}^{-}$ & $-0,045$ & $-0,012$ & 0,091 & $-0,099$ & $\begin{array}{c}, 430 * \\
*\end{array}$ & 0,065 &,$- 198^{*}$ & ,324** &,$- 201^{*}$ &,$- 275^{* *}$ &, $275^{* *}$ & 1 & & & & & & & \\
\hline TDESEMP & $-0,096$ & 0,026 & $-0,014$ & $-0,13$ & 0,038 & $-0,08$ & 0,045 & 0,106 & $-0,029$ & 0,106 & $-0,088$ & $-0,119$ & 0,024 & $-0,141$ &, $230 * *$ &,$- 230 * *$ & 0,099 & 1 & & & & & & \\
\hline IDHM & $-0,125$ & 0,027 & $-0,128$ & 0,142 & $\begin{array}{c}, 397^{*} \\
*\end{array}$ & $-0,094$ & $-0,122$ & 0,005 & $\begin{array}{c}, 222^{*} \\
*\end{array}$ & $\begin{array}{c}770^{*} \\
*\end{array}$ & 0,159 &, $227^{* *}$ &, $457^{* *}$ & ,183* & ,646** &,$- 646^{* *}$ &, $305^{*}$ & 0,067 & 1 & & & & & \\
\hline GINI &,$- 174^{*}$ & 0,053 & 0,163 & 0,152 & 0,105 & 0,02 & $-0,027$ & 0,025 & $-0,043$ & 0,128 &, $233^{* *}$ & $-0,033$ & 0,017 & $-0,126$ &,$- 214^{*}$ & ,214* & 0,049 & $-0,14$ & 0,143 & 1 & & & & \\
\hline SANINAD & $-0,08$ & $\begin{array}{c}- \\
0,072\end{array}$ & , 266** & 0,04 & 0,131 & 0,007 & 0,094 & 0,162 & $-0,125$ & $\begin{array}{c}500 * \\
*\end{array}$ & 0,064 & $-0,125$ & ,340** &,$- 274^{* *}$ &,$- 475^{* *}$ &, $475^{* *}$ & $\begin{array}{c}318^{*} \\
*\end{array}$ & $-0,064$ & $\begin{array}{c}433^{*} \\
*\end{array}$ & $\begin{array}{c}, 236^{*} \\
*\end{array}$ & 1 & & & \\
\hline
\end{tabular}




\begin{tabular}{|c|c|c|c|c|c|c|c|c|c|c|c|c|c|c|c|c|c|c|c|c|c|c|c|c|}
\hline TOBNAT & $-0,15$ & 0,08 & $-0,021$ & $-0,094$ & 0,027 & 0,05 & $-0,062$ & 0,085 & -169* & $\begin{array}{c}, 223^{*} \\
*\end{array}$ & -,185* &,$- 331^{* *}$ & ${ }^{-, 193 *}$ & $-0,145$ & 0,135 & $-0,135$ & $\begin{array}{c}, 262^{*} \\
*\end{array}$ &, $312^{* *}$ & $-0,02$ & $\begin{array}{c}- \\
0,149\end{array}$ & -,193* & 1 & & \\
\hline TXOBVIOL & ,203* & $\begin{array}{c}- \\
0,152\end{array}$ & $-0,029$ & 0,004 & $-0,04$ & $-0,008$ & 0,055 & $-0,118$ & $\begin{array}{c}218^{*} \\
*\end{array}$ & ,190* & 0,055 &, $338^{* *}$ & 0,128 &, $227^{* *}$ & $-0,143$ & 0,143 & $\begin{array}{c}312^{*} \\
*\end{array}$ &,$- 330 * *$ & 0,004 & 0,048 & 0,138 & $\stackrel{-}{-}, 900^{* *}$ & 1 & \\
\hline IFDM & 0,001 & $-0,08$ & $-0,076$ & 0,054 & $\begin{array}{c}, 233^{*} \\
*\end{array}$ & 0,057 & $\tilde{-}^{-} 216^{* *}$ & 0,017 & $\begin{array}{c}, 383^{*} \\
*\end{array}$ & $\begin{array}{c}, 437^{*} \\
*\end{array}$ & $-0,039$ & 0,12 & ,291** & ,308** & ,345** &,$- 345^{* *}$ & $\begin{array}{c}- \\
, 245^{*}\end{array}$ & $-0,041$ & $\begin{array}{c}, 443^{*} \\
*\end{array}$ & $\begin{array}{c}- \\
0,152\end{array}$ & ${ }^{-, 182^{*}}$ & $-0,118$ & ,181* & 1 \\
\hline
\end{tabular}

$* * p<0,01$

$* p<0.05$

QLAGRO: nível de especialidade na atividade agropecuária dos municípios de Mato Grosso; TXDESF: Taxa de Desflorestamento; TXHIDROG: área correspondente a hidrografia; CAGROTO: Consumo de defensivos agrícolas; IMPOS: impostos, líquidos de subsídios, sobre produtos, a preços correntes (R\$ 1.000); TEAGROP: número de empresas da agricultura, pecuária, produção florestal, pesca e aquicultura; TINDEXT: taxa de empresas indústrias extrativas; TEAGESG: taxa de empresas água, esgoto, atividades de gestão de resíduos e descontaminação; TETRNP: proporção de empresas ligadas ao setor de transporte, armazenagem e correio; RSMED: renda média domiciliar per capita; NCRURAL: número de estabelecimentos que obtiveram acesso ao crédito rural; VCRURAL: valor dos financiamentos agropecuários obtidos (mil reais); MAQIMP: número de máquinas e implementos agrícolas existentes nos estabelecimentos agropecuários; TUQTRANSP: taxa de utilização de meios de transportes; TPOPURB: população residente urbana; TPOPRUR: população residente rural; ANALFA: nível de instrução da pessoa que dirige o estabelecimento - analfabeto; TDESEMP: taxa de desemprego: percentual da população de 16 anos e mais, economicamente ativa, desocupada; IDHM: o índice de desenvolvimento humano municipal é composto de indicadores de três dimensões do desenvolvimento humano: longevidade, educação e renda; GINI: Índice Gini; SANINAD: proporção de domicílios com saneamento inadequado; TOBNAT: taxa de óbitos Naturais; TOBVIOL: taxa de óbitos Violentos; e IFDM: evolução do índice firjan geral.

Fonte: resultados da pesquisa (2020). 
A tabela 3, mostra os resultados do modelo de análise de regressão espacial final. 0 consumo de defensivos agrícolas, os impostos, o nível de instrução da pessoa que dirige o estabelecimento - analfabeto, e o índice Gini apresentaram associação inversa significativa com o nível de especialidade na atividade agropecuária (QLagro) dos municípios matogrossenses no período de 2005 a 2014 ( $p<0,05)$. Além disso, a taxa de empresas de transportes e a taxa de população residente rural foram positivamente associadas ao QLagro $(p<0,05)$.

Tabela 3 - Resultado da Regressão Múltipla Espacial.

\begin{tabular}{lllll}
\hline Variável & Coeficiente & Erro & t-estatístico & Probabilidade \\
\hline CONSTANT & 2,73976 & 0,559664 & 4,89536 & 0,00000 \\
CAGROTO & $-0,001273720$ & 0,000376 & $-3,39189$ & 0,00091 \\
IMPOS & $-0,000000827702$ & 0,0000003368720 & $-2,45702$ & 0,01529 \\
TETRNP & 6,084820000 & 1,919550 & 3,16993 & 0,00189 \\
TPOPRUR & 1,676380000 & 0,383382 & 4,37262 & 0,00002 \\
ANALFA & $-5,297240000$ & 1,212620 & $-4,36843$ & 0,00002 \\
GINI & $-2,523680000$ & 1,114020 & $-2,26538$ & 0,02509 \\
\hline
\end{tabular}

Fonte: resultados da pesquisa (2020).

\section{Discussão}

Primeiramente, foi determinada a média da participação percentual do PIB agropecuário dos 141 municípios do Estado de Mato Grosso no período de 2005-2014. Desse modo, foi possível identificar o nível de especialização de cada um desses municípios (Figura 2). Importante salientar, que os municípios identificados como especializados na atividade agropecuária, são relativamente novos, entre 20 e 80 anos de emancipação. A idade recente desses municípios indica que o processo de territorialização agrícola em Mato Grosso é relativamente recente. Entretanto, já possuem uma produção significativa de grãos (soja, milho, algodão e arroz) e na pecuária (bovinos, ovinos, caprinos e avicultura).

Em um segundo momento foi realizada a análise da distribuição espacial conjunta do QLagro (Figuras 3 e 4). Ao analisar as características dos municípios pertencentes ao agrupamento classificado como High-high, observa-se que são cidades relativamente novas, que se emanciparam a partir da década de 1930. Além disso, possuem um IDHM que indica um elevado grau de desenvolvimento humano. Outra característica em comum entre eles é 
o Índice Gini, que retrata uma relativa situação de igualdade na distribuição da renda municipal. Ademais, a base econômica desses municípios está voltada para a produtividade e escala de produção na pecuária intensiva de cria, recria e de corte; na agricultura, com culturas de soja, milho e arroz; e também, há extrativismo mineral. Embora sejam municípios especializados na produção latifundiária a maior parte de suas receitas ainda é oriunda de fontes externas (IBGE, 2020a).

Por outro lado, ao investigar as características dos municípios pertencentes ao agrupamento Low-low, identificou-se que a base econômica deste conglomerado se apoia na pecuária e em uma agricultura majoritariamente de subsistência nos inúmeros minifúndios, onde são desenvolvidas diversas culturas, com destaque para mandioca, banana, soja, milho, canade-açúcar (IBGE, 2020a). Nesse sentido, foi possível observar que a agricultura de subsistência é uma peculiaridade desse agrupamento classificado como "baixo-baixo" (Lowlow), mesmo assim, possui elevado grau de desenvolvimento e relativa situação de igualdade na distribuição da renda municipal (IBGE, 2020a). Entretanto, a renda geral tende a ser mais baixa, considerando o PIB per capita e o salário funcional dos municípios deste aglomerado (IBGE, 2020a).

Ao analisar as variáveis resultantes do modelo de análise de regressão espacial final (Tabela 3), observa-se que proporção de empresas ligadas ao setor de transporte, armazenagem e correio foi associada positivamente ao grau de especialidade na atividade agropecuária (QLagro). Nesse sentido, a existência de empresas de transporte favorece a especialização da atividade agropecuária, fortalecendo e tornando os municípios mais especializados no ramo do agronegócio. Estudos confirmam que o sistema de transportes contribui para a integralidade da cadeia de suprimentos e rende-se aos objetivos agregados, vinculados aos níveis regional ou industrial (Nielsen, Jespersen, Petersen, \& Hansen, 2003).

A proporção da população residente na área rural foi associada positivamente com o QLagro no modelo espacial final (Tabela 3). Constatou-se que a presença da população rural auxilia fortemente a especialidade do município na atividade agropecuária. Sendo assim, as políticas voltadas ao processo de desenvolvimento rural devem ser instrumentos de produtividade e ao mesmo tempo de conservação de um tecido social harmonizado e articulado no meio rural, não sendo orientadas apenas para o atendimento de necessidades de produtores modernos e viáveis (Balsadi, 2001). 
A variável correspondente aos impostos apresentou associação negativa neste estudo. Observou-se que a incidência de impostos não corrobora para com o QLagro (Tabela 3). Estudos afirmam que a incidência efetiva de impostos diverge daquela apoiada pelos formuladores de políticas econômicas, por entenderem que o governo tributa intensamente e indiretamente fatos geradores aos quais se intenciona isentar ou tributar de maneira superficial (Siqueira, Nogueira, \& Souza, 2001). A exemplo do caso da isenção de alguns impostos sobre as exportações de commodities brasileiras, implicitamente há tributação compensatória sobre o consumo de combustíveis e insumos de produção desses bens ou serviços exportados, entre outros. Entretanto, o ICMS dos insumos, por exemplo, pode ser utilizado como crédito no momento da exportação. Mesmo assim, o Brasil tem praticado políticas tarifárias prejudiciais a atividade agropecuária na proporção de $30 \%$ sobre o valor da produção agrícola (Simões, 1993).

A variável correspondente ao nível de instrução da pessoa que dirige o estabelecimento (analfabeto) foi associada negativamente a variável dependente da pesquisa (Tabela 3). Nesse sentido, o estado educacional de analfabetismo na população analisada não favorece o nível de especialização agropecuária. O analfabetismo é um tema muito discutido na problemática social, porém não se chega a uma solução universal (Llomovatte, 1989). As desigualdades regionais no âmbito da educação estão propensas a agravar ainda mais se forem deixadas ao arbítrio da lei inexorável do mercado (Ferraro \& Kreidlow, 2004). Portanto, a sociedade precisa realizar ações de alfabetização em adultos essencialmente para ser capaz de integrá-los em um nível superior de desenvolvimento e produção, diminuindo a segregação em meio à sociedade e transformando essas pessoas em força de trabalho com alto nível de conhecimento (Pinto, 2005).

O índice Gini de concentração de renda apresentou associação negativa à variável dependente do estudo (Tabela 3). Portanto, observou-se que concentração de renda prejudica e enfraquece a especialização agropecuária. Em geral, os países latinoamericanos, sobretudo o Brasil, destacam-se pela proeminente desigualdade na distribuição de renda (Székely \& Hilgert, 2007). Nesse sentido, alguns estudos afirmam que o crescimento econômico pode ser um aliado importante no combate da pobreza e da desigualdade (Bonelli \& Ramos, 1993). Portanto, vale considerar que a ação de reduzir a desigualdade é instrumento indispensável para redução da pobreza (Hoffmann, 2001). Se a 
pobreza for reduzida respeitando os limites ecossistêmicos, a qualidade de vida humana será beneficiada, a população será favorecida e ao mesmo tempo as pessoas serão instrumentos do processo de desenvolvimento sustentável (Assis, 2006). Principalmente, pelas populações mais pobres, tais como as envolvidas com o campo e a agricultura familiar, serem simultaneamente agentes da degradação e as mais atingidas pela degradação ambiental, essencialmente pela ausência de informação e recursos (Assis, 2006).

O consumo de defensivos agrícolas foi associado negativamente no modelo espacial final (Tabela 3). Evidenciou-se que o consumo de defensivos agrícolas desfavorece a especialização da atividade agropecuária para o período em análise. De acordo com o boletim anual de vendas do Instituto Brasileiro do Meio Ambiente e dos Recursos Naturais Renováveis (IBAMA, 2014), Mato Grosso lidera o consumo de defensivos agrícolas no cenário nacional principalmente por ser o maior estado produtor de soja, milho e algodão herbáceo no país, segundo maior produtor de arroz e quarto maior produtor de cana-deaçúcar (IBGE, 2020). Esse situação é consequência da transformação do alimento em commoditie e da transfiguração do mesmo em combustível (produção de energia) (Bombardi, 2012). Desse modo, esta "comoditização" reflete na saúde pública (Pignati et al., 2017), pois há alta incidência de intoxicação tanto na aplicação direta de defensivos agrícolas quanto no consumo de alimentos envenenados (Soares \& Porto, 2012). Além disso, as discrepâncias regionais na produção agrícola acompanham um processo tecnológico e de incentivos fiscais de exploração e uso da terra que seguem coexistindo com as fragilidades nas legislações ambientais, controle social e com políticas que favorecem este modelo químico-dependente (Pignati et al., 2017). Portanto, estudos afirmam que uma intervenção, por meio de programas de incentivo a agricultura orgânica e outras políticas públicas, poderiam ser utilizadas como maneira de reverter esse ataque a saúde e a sociedade (Soares \& Porto, 2012).

Observa-se então que a dialética econômico-sócio-ambiental do agronegócio encontrada é sustentada por um modelo agroquímico-dependente consolidado que desfavorece a sustentabilidade e o desenvolvimento da especialização agropecuária, afetando a saúde pública, bem como, o meio ambiente. Nesse sentido, a solução para esta dicotomia se encontra na teoria prática do desenvolvimento de Ignacy Sachs: por meio de uma 
ecossocioeconomia é possível associar ao sucesso econômico o incremento democrático do bem-estar social e a conservação ambiental (Sachs, 2007).

\section{Conclusão}

Os resultados do presente estudo evidenciaram uma distribuição espacial conjunta bastante desigual do nível de especialização na atividade agropecuária dos municípios no estado de Mato Grosso, para o período de 2005-2014. Ocorreu uma predominância de agrupamentos espaciais significativos do QLagro nas regiões Central e Sudeste do estado.

A distribuição espacial do QLagro apresentou-se aleatória, com significativa autocorrelação espacial, na aplicação da Estatística de Moran I Global. Foi possível observar autocorrelações espaciais do tipo "alto-alto", indicando agrupamento com municípios caracterizados por terem a maior parte de suas receitas provenientes de fontes externas e suas atividades estão voltadas a produtividade e escala de produção. Foi possível observar também, que a agricultura de subsistência é uma peculiaridade do agrupamento identificado como "baixobaixo" (Low-low). Além disso, apresentam índices de desenvolvimento municipais e de distribuição de renda inferiores ao grupo identificado como "alto-alto" (High-high).

Por meio da análise do Índice de Moran Local, foi possível observar que existem externalidades multidirecionais, relacionadas ao nível de especialidade na atividade agropecuária para os anos de 2005 a 2014, representada pelos municípios de Primavera do Leste $(p=0,001)$, Acorizal, Rondolândia e Paranatinga $(p=0,01)$, refletindo áreas que apresentam dinâmica espacial própria e correlação local significativamente divergente de outras regiões do estudo.

As variáveis relacionadas a proporção de empresas ligadas ao setor de transporte, a proporção da população residente na área rural, os impostos, o nível de instrução da pessoa que dirige o estabelecimento - analfabeto, o índice Gini de concentração de renda e o consumo de defensivos agrícolas, permaneceram correlacionadas ao nível de especialidade na atividade agropecuária no modelo final da análise de regressão múltipla espacial. Sem dúvida, o resultado mais expressivo desta pesquisa envolve a evidência de que o consumo 
de defensivos agrícolas desfavorece a especialização da atividade agropecuária no contexto mato-grossense.

O produto desta pesquisa revela a importância em se analisar os fatores associados aos agrupamentos espaciais do setor agropecuário e seus resultados trazem importantes contribuições para a compreensão dos aspectos ecossocioeconômicos espaciais envolvidos neste processo. Além de auxiliar na visibilidade desses fatores que impactam de maneira significativa, tanto positiva quanto negativamente.

Visto que os resultados sugerem que a Especialização Agropecuária (QLagro), nos municípios ao sul da Amazônia Legal, não possuem uma ecossocioeconomia, sugere-se a necessidade de redirecionamento das políticas públicas brasileiras destinadas ao desenvolvimento sustentável, na contribuição para o setor no presente e na garantia de funcionamento ecossistêmico do mesmo para o futuro.

Ao avaliar a distribuição do nível de especialização agropecuária e sua relação com as variáveis de dimensões econômicas, sociodemográficas e ambientais foi possível observar a dimensão da pesquisa a partir da constatação de uma generalização do nível de especialização por meio da análise do espaço geográfico. Percebe-se que o estudo não é terminativo, portanto, sugere-se sua continuidade mediante o aprofundamento das análises em regiões especificas, dada à intenção de entender os fundamentos encontrados nos resultados que trazem importantes contribuições para a compreensão dos aspectos espaciais, econômicos, sociodemográficos e ambientais envolvidos neste processo de especialização regional que proporcionou informações essenciais de planejamento futuro que vise uma estratégia competitiva de desenvolvimento voltado para regiões menos desenvolvidas.

\section{Referências}

Almeida, E. S. de. (2004). Curso de econometria espacial aplicada. Retrieved December 8, 2016, from http://docslide.com.br/documents/apostila-econometria-espacial.html\#.WEmIWhwicD4.mendeley

Anselin, L. (1995). New Directions in Spatial Econometrics (L. Anselin \& R. J. G. M. Florax, Eds.). Berlin, Heidelberg: Springer Berlin Heidelberg. https://doi.org/10.1007/978-3-642-79877-1

Anselin, L., \& Rey, S. J. (2010). Perspectives on Spatial Data Analysis (L. Anselin \& S. J. Rey, Eds.). Berlin, Heidelberg: Springer Berlin Heidelberg. https://doi.org/10.1007/978-3-642-01976-0

Assis, R. L. de. (2006). Desenvolvimento rural sustentável no Brasil: perspectivas a partir da integração de 
ações públicas e privadas com base na agroecologia. Economia Aplicada, 10(1), $75-89$. https://doi.org/10.1590/S1413-80502006000100005

Balsadi, O. V. (2001). MUDANÇAS NO MEIO RURAL E DESAFIOS PARA O DESENVOLVIMENTO SUSTENTÁVEL. São Paulo Em Perspectiva, 15(1), 155-165. https://doi.org/10.1590/S0102-88392001000100017

Bombardi, L. M. (2012). Agrotóxicos e agronegócio: arcaico e moderno se fundem no campo brasileiro. Direitos Humanos No Brasil 2012, 75-86.

Bonelli, R., \& Ramos, L. R. A. (1993). Distribuição de renda no Brasil: avaliação das tendências de longo prazo e mudanças na desigualdade desde meados dos anos 70. Revista de Economia Política, Vol. 13, pp. 76-97. Retrieved from http://www.rep.org.br/pdf/50-5.pdf

Cavalcanti, C. (2010). Concepções da economia ecológica: suas relações com a economia dominante e a economia ambiental. Estudos Avançados, 24(68), 53-67. https://doi.org/10.1590/S0103-40142010000100007

Celemín, J. P. (2009). Autocorrelación espacial e indicadores locales de asociación espacial. Importancia, estructura y aplicación Spatial Autocorrelation and Local Indicators of Spatial Association: Importance, Structure and Application. Revista Universitaria de Geografía, 18, 11-31. Retrieved from https://www.redalyc.org/articulo.oa?id=3832/383239099001

Crocco, M. A., Galinari, R., Santos, F., Lemos, M. B., \& Simões, R. (2006). Metodologia de identificação de aglomerações produtivas locais. Nova Economiaova Economia_Belo Horizonte, 16(2), $211-241$. https://doi.org/10.1590/S0103-63512006000200001

Ferraro, A. R., \& Kreidlow, D. (2004). Analfabetismo no Brasil: configuração e gênese das desigualdades regionais. Educação e Realidade, 29(2), 179-200.

Haddad, P. R. (1989). Medidas de localização e de especialização. In Economia Regional: Teorias e Métodos de Análise (pp. 67-206). Fortaleza: BNB/ETENE.

Hoffmann, R. (2001). Distribuição de renda e crescimento econômico. Estudos Avançados, 15(41), 67-76. https://doi.org/10.1590/S0103-40142001000100007

IBAMA. Instituto Brasileiro do Meio Ambiente e dos Recursos Naturais Renováveis. (2014). Boletins Anuais: vendas por UF (R. M. Rebelo, R. A. Vasconcelos, B. D. M. C. Buys, J. A. Rezende, K. de O. C. de Moraes, \& R. de P. Oliveira, Eds.).

IBGE. (2020a). Instituto Brasileiro de Geografia e Estatística: Cidades@. Retrieved February 4, 2020, from Instituto Brasileiro de Geografia e Estatística website: http://cidades.ibge.gov.br/xtras/perfil.php?codmun=510790

IBGE. (2020b). Sistema de Contas Nacionais: Produto Interno Bruto dos Municípios 2008-2017. Retrieved July 12, 2020, from Instituto Brasileiro de Geografia e Estatística website: https://sidra.ibge.gov.br/pesquisa/pibmunic/tabelas

Lara, F. M., Fiori, T. P., \& Zanin, V. (2010). Notas sobre medidas de concentração e especialização: um exercício preliminar para o emprego no Rio Grande do Sul. Textos Para Discussão FEE, (83). Retrieved from https://www.fee.rs.gov.br/tedes/notas-sobre-medidas-de-concentracao-e-especializacao-um-exerciciopreliminar-para-o-emprego-no-rio-grande-do-sul/

Lins, J. G. M. G., \& Da Silva, M. V. B. (2017). Identificação e análise espacial de concentração dos clusters produtivos dos setores de confecções e couro-calçadista na região Nordeste entre 1997 e 2012. Revista Economia Ensaios, 31(2). https://doi.org/10.14393/REE-v31n2a2017-7

Llomovatte, S. (1989). Analfabetismo en Argentina. Buenos Aires: Niño y Dávila.

Marafon, G. J., \& Lima, V. M. de S. (2018). Turismo rural: resgatando conceitos e atualizando reflexões. In G. J. Marafon, L. Q. Arias, \& M. A. Sánchez (Eds.), Estudos territoriais no Brasil e na Costa Rica (1st ed., pp. 91-111). Rio de Janeiro: EDUERJ. https://doi.org/10.7476/9788575114995.0005

Moi, G. P., Silva, A. M. C., Galvão, N. D., de Castro Meneghim, M., \& Pereira, A. C. (2018). Spatial analysis of the death associated factors due oral cancer in Brazil: an ecological study. BMC Oral Health, 18(1), 14. https://doi.org/10.1186/s12903-018-0473-y 
Nielsen, L. D., Jespersen, P. H., Petersen, T., \& Hansen, L. G. (2003). Freight transport growth-a theoretical and methodological framework. European Journal of Operational Research, 144, 295-305. https://doi.org/10.1016/S0377-2217(02)00395-8

Pignati, W. A., Lima, F. A. N. de S. e, Lara, S. S. de, Correa, M. L. M., Barbosa, J. R., Leão, L. H. da C., \& Pignatti, M. G. (2017). Distribuição espacial do uso de agrotóxicos no Brasil: uma ferramenta para a Vigilância em Saúde. Ciência \& Saúde Coletiva, 22(10), 3281-3293. https://doi.org/10.1590/1413-812320172210.17742017

Pinto, Á. (2005). Sete lições sobre educação de adultos. São Paulo: Cortez.

Sachs, I. (2007). Rumo a ecossocioeconomia: teoria prática do desenvolvimento (P. F. Vieira, Ed.). São Paulo: Cortez.

Santos, C. do. (2015). O espírito do capitalismo na ocupação dos cerrados brasileiros nos estados da Bahia e do Piauí. GOT - Geography and Spatial Planning Journal, (8), 229-253. https://doi.org/10.17127/got/2015.8.012

Simões, R. (1993). As grandes questões do desenvolvimento agrícola e do desenvolvimento rural. Congresso Brasileiro de Economia e Sociologia Rural - SOBER, 942-956. Brasília, DF: SOBER. Retrieved from http://www.iea.sp.gov.br/out/publicacoes/pdf/asp-2-03-2.pdf

Siqueira, R. B. de, Nogueira, J. R., \& Souza, E. S. de. (2001). A incidência final dos impostos indiretos no Brasil: efeitos da tributação de insumos. Revista Brasileira de Economia, 55(4), 513-544. https://doi.org/10.1590/S0034-71402001000400004

Soares, W. L., \& Porto, M. F. de S. (2012). Uso de agrotóxicos e impactos econômicos sobre a saúde. Rev Saúde Pública, 46(2), 209-217. https://doi.org/10.1590/S0034-89102012005000006

Székely, M., \& Hilgert, M. (2007). What's behind the inequality we measure? An investigation using Latin American data. Oxford Development Studies, 35(2), 197-217. https://doi.org/10.1080/13600810701427626

Taylor, R. (1990). Interpretation of the Correlation Coefficient: A Basic Review. Journal of Diagnostic Medical Sonography. https://doi.org/10.1177/875647939000600106

Veiga, J. E. da. (2010). Indicadores de Sustentabilidade. Estudos Avançados, 24(68), 39-52. https://doi.org/10.1590/S0103-40142010000100006 